\title{
Development and validation of a high-performance liquid chromatography method for the quantitation of intracellular PARP inhibitor Olaparib in cancer cells.
}

Pierre Daumar ${ }^{*, a}$, Robin Dufour ${ }^{a, b}$, Clémence Dubois ${ }^{a, b}$, Frédérique Penault-Llorca ${ }^{a, b}$, Mahchid Bamdad ${ }^{a}$ \& Emmanuelle Mounetou ${ }^{a}$

a Université Clermont Auvergne, Institut Universitaire de Technologie, INSERM, U1240 Imagerie Moléculaire et Stratégies Théranostiques, F-63000 Clermont Ferrand, France

b Université Clermont Auvergne, Centre Jean Perrin, INSERM, U1240 Imagerie Moléculaire et Stratégies Théranostiques, F-63000 Clermont Ferrand, France

\section{*Corresponding Author}

Pierre Daumar

Address: Institut Universitaire de Technologie - Département Génie Biologique, Université d'Auvergne, 5 avenue Blaise Pascal, BP 86 - 63172 AUBIERE CEDEX France

Email: pierre.daumar@uca.fr

Phone: +33473177061 


\begin{abstract}
Olaparib is a potent PARP inhibitor in clinical use for cancer therapy. A bioanalytical assay was developed and validated for quantitation of intracellular level of olaparib in cells exposed to the drug. The assay involves an optimized and straightforward sample pretreatment with acetonitrile for olaparib solubilization, cell lysis and protein precipitation, and a high performance liquid chromatography (HPLC) method with ultraviolet detection. Several parameters in both the sample preparation and the detection steps were investigated. Optimal chromatographic conditions were achieved with a $5 \mu \mathrm{L}$ injection on a Nova-Pak ${ }^{\circledR} \mathrm{C}_{18}$ column $(150 \times 3.9 \mathrm{~mm}, 4$ $\mu \mathrm{m})$ using a mobile phase consisting of acetonitrile and ultra-pure water in gradient mode, at a constant 1.2 $\mathrm{mL} / \mathrm{min}$ flow rate, at $35^{\circ} \mathrm{C}$. Detection was carried out at $254 \mathrm{~nm}$ and a diode array detector was used to insure purity of the olaparib peak. The method was validated according to Food and Drug Administration guidelines. Linearity, accuracy and precisions were satisfactory over the concentration range of $200-2000 \mathrm{ng} / \mathrm{mL}$. Limits of detection and quantification for olaparib were $50 \mathrm{ng} / \mathrm{mL}$ and $200 \mathrm{ng} / \mathrm{mL}$, respectively. Good stability was showed in three relevant analytical conditions. Finally, the validated analytical method was successfully used to estimate the intracellular level of olaparib in SUM1315 breast cancer cells. A significant difference was observed in intracellular drug level after 1 and 3 hour incubations. This method permitting measurement of drug level in tumor cells would allow dosage optimization and improvement of treatment response predictions.
\end{abstract}

\title{
Keywords
}

HPLC-UV-DAD · bioanalytical method validation · intracellular drug concentration • PARP inhibitors • Olaparib

\section{Introduction}

Over the last decade, the inhibition of poly (ADP-ribose) polymerase (PARP), a nuclear enzyme involved in many processes including DNA repair and cell death, has become a promising personalized therapeutic strategy in cancer treatment [1-4]. PARP inhibitors, a new class of antineoplastic drugs, can induce tumor-specific synthetic lethality in cancer cells with defective DNA damage repair system, like those associated to mutations of the BRCA tumor suppressor genes [5-8]. Olaparib (AZD2281, Figure 1), veliparib (ABT-888), niraparib (MK4827) or rucaparib (AG014699) are examples of potent PARP inhibitors having recently reached advanced clinical trials as combination and/or standalone targeted therapies, especially in breast and ovarian cancers. Olaparib (Lynparza) was the first to gain the European Commission (2014) and the United States Food and Drug Administration (2015) regulatory approvals for use in patients with $B R C A$-mutated ovarian cancer $[5,9,10]$.

PARP inhibitors have great therapeutic potential and are anticipated to have broad clinical application in future cancer therapy [10]. However, both preclinical and clinical studies have revealed that tumor cells sensitivity towards PARP inhibitors can vary widely, and that treatments efficacy needs optimization. PARP being an intracellular target, the sensitivity of tumor cells and the effectiveness of a PARP targeted therapy are influenced 
by a key factor, namely the amount of PARP inhibitors reaching the intracellular compartment [11]. As for any drug with an intracellular target, the disposition of PARP inhibitors is greatly affected by processes such as elimination, metabolism, drug uptake, and expression/upregulation of transmembrane drug efflux transporters. The latter, particularly relevant for PARP inhibitors, was identified during early preclinical studies as a major resistance mechanism $[3,12,13,14]$.

In this context, methods that provide measurements of PARP inhibitors concentration, especially in tumor cells, have potential for drug dosage optimization and improvement of drug response predictions. Overall, such data would lead to the implemented therapeutic potential of PARP inhibitors. Therefore, a reliable methodology to assess intracellular level of PARP inhibitors in cancer cells is warranted, and could ultimately provide a mean to assess relationships between PARP inhibitors intracellular level and treatment efficacy.

Measuring intracellular concentration of a drug is challenging and has relied most of the time on the use of radiolabeled analogues. However, the main limitation of this methodology originates in the fact that such compounds are not readily available $[15,16]$. Mass spectrometry is a very sensitive and specific technique to determine drug levels in cells [16,17]. Accordingly, liquid chromatography-tandem mass spectrometry (LCMS/MS) is the method of choice for low drug level detection but still requires high-cost instruments and timeconsuming sample preparations. Regarding PARP inhibitors, LC-MS/MS methods were only reported for quantitative measurements in cell culture medium, human plasma, tumor and brain homogenates [19-23]. Oplustilova et al. recently mentioned the intracellular level determination of an olaparib analog by LC-MS but no technical details were given on the analytical method that was employed [24]. Finally, flow cytometric analysis was used recently to perform single-cell analysis and assess intracellular concentration of fluorescent drugs $[11,18,25,26]$. Although promising, the development of this technique is still at an early stage and the low number of drugs with intrinsic fluorescence represents a major limitation. Reversed-phase high performance liquid chromatography (HPLC) with Ultraviolet Diode Array Detector (UV-DAD) analyses allow for sensitive quantitation of many compounds with accessible equipment. However, to the best of our knowledge, no validated method based on this technique for the determination of intracellular drug was ever reported in the literature.

The aim of the present study was to develop a simple, sensitive and reliable HPLC-UV-DAD procedure for quantitation of intracellular PARP inhibitor levels. The assay provides the advantage of working with un-labeled compounds and includes a straightforward and reliable cell sample pretreatment developed by varying the nature and the volume of the solvent used for solubilization of intracellular drug. The optimized analytical method was validated according to international guidelines for bioanalytical method validation and applied to the estimation of olaparib intracellular level in SUM1315 breast cancer cells.

\section{Material and Methods}

\subsection{Chemicals and Reagents}


Olaparib was purchased from Sequoia Research Products (Pangbourne, UK). All chemicals were acquired from Sigma-Aldrich (France). Dimethyl sulfoxide (DMSO) was of molecular biology grade. Water and acetonitrile were of HPLC gradient grade (VWR Chemicals, France).

\subsection{SUM1315 cell culture}

SUM1315 cells were obtained from Asterand (Royston, UK) and maintained by weekly serial passage in a 5\% $\mathrm{CO}_{2}$ atmosphere at $37^{\circ} \mathrm{C}$. Cells were cultured in Ham's F12 medium (GIBCO ${ }^{\mathrm{TM}}$, France) supplemented with 5\% fetal bovine serum, $10 \mathrm{mM}$ HEPES buffer, $20 \mu \mathrm{g} / \mathrm{mL}$ gentamicin, $10 \mathrm{ng} / \mathrm{mL}$ EGF and $4 \mu \mathrm{g} / \mathrm{mL}$ insulin [26].

\subsection{Stock solutions, calibration standards and quality control samples}

A $1 \mathrm{mg} / \mathrm{mL}$ stock solution of olaparib was prepared by dissolving an appropriate amount of olaparib in DMSO. By dilution of this solution with acetonitrile, separate $10000 \mathrm{ng} / \mathrm{mL}$ stock solutions for calibration standards and quality control samples (QC samples) were prepared and stored at $-20{ }^{\circ} \mathrm{C}$. Both stock solutions were further diluted to obtain working solutions. Calibration standards were prepared daily before each validation run. These were prepared by adding the appropriate amount of the $10000 \mathrm{ng} / \mathrm{mL}$ stock solution to acetonitrile to obtain concentrations of 200, 400, 600, 800, 1000 and $2000 \mathrm{ng} / \mathrm{mL}$ for olaparib. QC samples were prepared in a similar manner to obtain olaparib spiked solutions in acetonitrile used for sample preparation. Final olaparib concentrations at QC-low, QC-mid and QC-high were 300, 800 and $1800 \mathrm{ng} / \mathrm{mL}$, respectively.

\subsection{Chromatographic equipment and conditions}

Analytical HPLC experiments were performed on a HP 1100 series LC system equipped with an online degasser, a quaternary pump, a column oven, a photodiode array detector (DAD) and an autosampler. Water and acetonitrile $(\mathrm{ACN})$ used for the preparation of the mobile phase were filtered through a $0.45 \mu \mathrm{m}$ Durapore Polyvinylidene difluoride (PVDF) membrane filter (Merck Millipore, UK) before being used in the chromatographic system. The temperature of the column oven was kept at $35^{\circ} \mathrm{C}$. A gradient elution was applied at a flow rate of $1.2 \mathrm{~mL} / \mathrm{min}$ through a reversed-phase Nova-Pak ${ }^{\circledR} 4 \mu \mathrm{m} \mathrm{C}_{18}$ column $(150 \times 3.9 \mathrm{~mm}$; Waters Corporation, Milford, MA, USA) with an additional Nova-Pak ${ }^{\circledR}$ guard column. The following gradient was employed: 0 - 13 min, 5 - 35\% ACN; 13 - 16 min, 35-95\% ACN; 16 - 21 min, 95\% ACN; 21 - 22 min, 95 $5 \%$ ACN; $22-28 \mathrm{~min}, 5 \%$. A sample volume of $5 \mu \mathrm{L}$ was injected. UV spectra were recorded from 210 to 400 $\mathrm{nm}$ from 0 to $14 \mathrm{~min}$ and the monitoring wavelength for olaparib was set to $254 \mathrm{~nm}$. Agilent ChemStation software (version B.04.03-SP1, Agilent Technologies, France) was used for acquisition of chromatograms, spectra and integration data.

\subsection{Sample preparation}

SUM1315 cells were seeded at a density of 250000 cells $/ \mathrm{mL}$ in $5 \mathrm{~mL}$ of culture medium and incubated at $37^{\circ} \mathrm{C}$ during $24 \mathrm{~h}$ for cell adhesion to the flask. The culture medium was removed. Cells were subsequently washed with ice-cold PBS $(2 \times 2 \mathrm{~mL})$ and then lysed by scraping in acetonitrile $(2 \mathrm{~mL})$ in conjunction with sonication $(5$ $\times 3 \mathrm{~s})$. After centrifugation of the samples at $4{ }^{\circ} \mathrm{C}(350 \mathrm{~g}, 10 \mathrm{~min})$, the supernatant was filtered through a $0.45 \mu \mathrm{m}$ PVDF membrane filter and pipetted into a $1 \mathrm{~mL}$ autosampler vial for injection. 


\subsection{Bioanalytical method validation}

The described method was validated according to internationally accepted recommendations for bioanalytical method validation [28].

\subsubsection{Selectivity}

Six different cell samples processed without olaparib were analyzed for peaks of substances interfering with that of olaparib at $10.1 \mathrm{~min}$. The chromatograms were compared to those obtained with the $200 \mathrm{ng} / \mathrm{mL}$ calibration standards and to blank samples (HPLC gradient grade acetonitrile).

\subsubsection{Carry over effect}

Carry over was investigated by injecting a blank sample (HPLC gradient grade acetonitrile) after each QC-high sample injection in each analytical run.

\subsubsection{Recovery}

The recovery of olaparib over the whole process from cell samples was determined using QC samples (QC-low, QC-mid, QC-high) and olaparib standard solutions in acetonitrile at the three validation levels (QC-low, QCmid, QC-high). The recovery was calculated by comparing olaparib peak areas from QC samples $(n=12$ for each concentration level) submitted to sample preparation (cell scraping and sonication, centrifugation, filtration) with the corresponding mean peak area of the unprocessed standard solutions $(n=4)$.

\subsubsection{Calibration curves}

The 200 and $2000 \mathrm{ng} / \mathrm{mL}$ calibration standards were processed in duplicate for each daily calibration, whereas the levels in between were processed only once. Linear regression analysis was employed to define the calibration curves using the olaparib peak area versus the nominal concentration of the calibration standard $(\mathrm{ng} / \mathrm{mL})$. For each analytical run, the calibration curve was accepted if all calibration standards had a deviation within $\pm 15 \%$ of the nominal concentration. A deviation of $\pm 20 \%$ for the $200 \mathrm{ng} / \mathrm{mL}$ calibration standards was accepted. Subsequently, the determined linear regression formula was used to measure concentrations of QC and unknown cell samples after integration of the olaparib peak area on the corresponding chromatogram.

\subsubsection{Precision and accuracy}

QC samples were used to assess the precision and the accuracy of the method. Each QC sample was processed in a quintuple analysis in four analytical runs on four separate days. The relative standard deviation (RSD) was calculated to determine the within day precision (repeatability) and the between day precision (reproducibility). The deviation of the mean measured concentration from the nominal value in percentage (bias \%) was calculated to determine the within and between day accuracies.

\subsubsection{Limit of quantification and limit of detection}

The sensitivity was evaluated by determining the limit of quantification (LOQ), defined as the lowest concentration of the standard curve that can be measured with acceptable inter- and intra-day precisions and 
accuracies. RSD values lower than $20 \%$ and bias values within $\pm 20 \%$ were considered acceptable for precision and accuracy, respectively.

The limit of detection (LOD) is defined as the lowest concentration of a compound that an analytical process can reliably differentiate from background level. LOD was determined by visual evaluation of chromatograms of standards on which olaparib could be reliably detected.

\subsubsection{Stability}

The stability of olaparib in acetonitrile was investigated in QC-low and QC-high samples stored in HPLC glass vials. Quadruplicate analysis of these samples was performed after storage at room temperature $\left(20{ }^{\circ} \mathrm{C}\right)$ for $24 \mathrm{~h}$ after thawing, three freeze-thaw cycles (thawing at room temperature during $12 \mathrm{~h}$ and freezing again at $-20{ }^{\circ} \mathrm{C}$ for at least one day), and storage at $-20^{\circ} \mathrm{C}$ for one month.

\subsection{Method applicability: determination of intracellular concentrations of olaparib in SUM1315 cells}

SUM1315 cells were seeded at a density of 250000 cells $/ \mathrm{mL}$ in $5 \mathrm{~mL}$ of culture medium and incubated at $37^{\circ} \mathrm{C}$ during $24 \mathrm{~h}$ for cell adhesion to the flask. The cell cultures were then exposed to $50 \mu \mathrm{M}$ olaparib. For each experiment, the required volume of a $50 \mathrm{mM}$ olaparib stock solution was added so that the final DMSO concentration remained always constant i.e. $0.1 \%$. In parallel, untreated cells were cultured as control. The intracellular concentration of olaparib in SUM1315 cells was evaluated after 1 and $3 \mathrm{~h}$ of $50 \mu \mathrm{M}$ olaparib exposures. After each incubation time, the culture medium was removed. Cells were subsequently washed with ice-cold PBS $(2 \times 2 \mathrm{~mL})$ and then lysed by scraping in acetonitrile $(2 \mathrm{~mL})$ in conjunction with sonication $(5 \times$ $3 \mathrm{~s})$. After centrifugation of the samples at $4^{\circ} \mathrm{C}(350 \mathrm{~g}, 10 \mathrm{~min})$, the supernatant was filtered through a $0.45 \mu \mathrm{m}$ PVDF membrane filter and pipetted into a $1 \mathrm{~mL}$ autosampler vial for injection. The intracellular concentration of olaparib in cells was subsequently determined by HPLC-UV analysis. All experiments were performed in triplicate $(n=3)$ and each sample was analyzed three times. Statistical analyses were performed with Microsoft Excel using a two-sided Student's t test with $\mathrm{p}<0.05$ considered significant.

\section{Results and Discussion}

\subsection{Method development}

The aim of the present study was to develop and validate a simple and sensitive reversed-phase HPLC method with UV-DAD detection for quantitation of intracellular PARP inhibitor levels.

Starting from cells in culture flasks, the method reported herein relies on a straightforward sample pretreatment. For each cell sample, the culture medium was first removed and a wash step was performed in ice cold PBS in order to avoid disturbance in drug equilibrium. Indeed, the low temperature limits active transport of intracellular drug out of the cells during pretreatment. By analyzing the washing solution by HPLC, it was shown that a single wash was not sufficient to ensure the total elimination of the remaining extracellular drug in the culture medium and on the cell surface. For each sample, the wash was repeated twice with $2 \mathrm{~mL}$ of PBS, a volume which was kept minimal but was sufficient to cover the whole surface of the flask. 
With the goal of developing an assay with limited manipulations, the next step implied to identify an organic solvent or a solvent mixture adapted to (i) lyse the cells when associated to sonication, (ii) solubilize intracellular olaparib, and (iii) allow precipitated proteins and cell debris to be easily eliminated by centrifugation.

Acetonitrile and methanol were tested pure and as a 50/50 (v/v) mixture. A volume of $2 \mathrm{~mL}$ was chosen. Each trial gave a different result regarding cell structure. Indeed, after a very short time in contact with the cells, it was found by optical microscopy examination that pure methanol and the acetonitrile/methanol mixture led to fixation of the cells. Acetonitrile gave satisfactory results and was therefore chosen for this critical step. Subsequently, a simple filtration of the supernatant through PVDF membrane filters directly in HPLC vials provided the samples to be analyzed. A volume of $2 \mathrm{~mL}$ chosen after flask surface consideration allowed samples to be sufficiently concentrated for HPLC-UV detection, thus avoiding concentrating and reconstitution steps before analysis. HPLC injectable samples were obtained from cell cultures after a short time and a limited number of steps.

With regards to the chromatographic conditions, a usual UV-DAD detection system and standard chromatography elution conditions were employed with a general purpose Nova-Pak ${ }^{\circledR} \mathrm{C}_{18}$ column equipped with an additional Nova-Pak ${ }^{\circledR}$ guard column. A water-acetonitrile mobile phase with a gradient elution was tested. The gradient was optimized by varying the acetonitrile percentage in the initial mobile phase from 5 to $20 \%$ and the gradient slope. A low 5\% acetonitrile starting mobile phase with a slow increase to $35 \%$ over 13 minutes was necessary for olaparib to be sufficiently retained on the column while keeping a reasonably short retention time. The choice of the detection wavelength was crucial for the sensitivity and selectivity of the method. A compromise was found and the detection wavelength was set to $254 \mathrm{~nm}$, which corresponds to a maximum on the UV absorption spectrum of olaparib (Fig. 2). Although a better sensitivity could have been reached at a lower wavelength, too many peaks from co-eluting endogenous substances were observed when recording chromatograms at $210 \mathrm{~nm}$ for selectivity assessment.

The use of acetonitrile during the sample pretreatment offers the opportunity of a small volume direct injection in the chromatographic system. Initially, broad peaks were observed even with injection volumes as low as $5 \mu \mathrm{L}$. Therefore, the influence of two parameters was investigated to optimize peak shapes: the mobile phase flow rate was tested at 0.8 and $1.2 \mathrm{~mL} / \mathrm{min}$, and different temperatures of the column oven were applied, every $5^{\circ} \mathrm{C}$ from 25 to $45^{\circ} \mathrm{C}$. Eventually, sharp and symmetric peaks were obtained with the following optimal conditions: 1.2 $\mathrm{mL} / \mathrm{min}$ for the mobile phase flow rate and $35^{\circ} \mathrm{C}$ for the oven temperature. In these optimized chromatographic conditions, olaparib was detected at a retention time of $10.1 \mathrm{~min}$, for a $28 \mathrm{~min}$ total run time, including the column equilibration delay.

\subsection{Method validation}

\subsubsection{Selectivity and carry over effect}

Control SUM1315 cell samples processed without olaparib were analyzed under the chosen optimized chromatographic conditions to investigate the selectivity of the method. The chromatograms showed several peaks from endogenous substances but revealed no interference at the retention time of olaparib (10.1 min), indicating that the described method is selective. Typical HPLC chromatograms of a control cell sample processed without olaparib, a blank sample of gradient grade acetonitrile, a standard solution of olaparib in 
acetonitrile, and a QC-high sample are shown on figure 3a, b c and d, respectively. The control cell sample chromatogram (Fig. 3a) showed peaks of substances eluting before or after olaparib but no peak was observed at $10.1 \mathrm{~min}$. The blank sample chromatogram (Fig. 3b) showed no peak and a flat baseline, and finally a well resolved peak with symmetrical shape corresponding to olaparib appears at $10.1 \mathrm{~min}$ on both chromatograms $3 \mathrm{c}$ and 3d. For each sample in which olaparib was detected, the UV-DAD detector was used to assess the purity of the peak at $10.1 \mathrm{~min}$ by comparing the corresponding UV absorption spectrum $(210-400 \mathrm{~nm})$ with that obtained with a standard sample of olaparib. A perfect match was observed for each sample, which also indicates a good selectivity. A typical HPLC chromatogram of a cell sample treated with olaparib $50 \mu \mathrm{M}$ for $3 \mathrm{~h}$ is shown on Figure 4, with the corresponding well resolved peak appearing at $10.1 \mathrm{~min}$. No carry-over effect was observed when blank samples were analyzed after QC-high samples injections.

\subsubsection{Recovery}

The recoveries of QC samples over the sample preparation process were calculated as the $\%$ ratio of olaparib peak area from processed QC samples and unprocessed standard solutions in acetonitrile. Percentages are shown in Table 1. Recoveries were quantitative at QC-low, QC-mid and QC-high levels. As no analyte losses were involved in our sample preparation, the use of internal standard was not necessary. Moreover, representative chromatograms of a standard solution of olaparib in acetonitrile (Fig. 3c) and a QC-high sample (Fig. 3d) presented the same profile. These results proved the relevance of using olaparib standard solutions in acetonitrile to construct calibration curves.

\subsubsection{Calibration curves}

The linearity of the method was then evaluated. Olaparib standard solutions in acetonitrile over the 200-2000 $\mathrm{ng} / \mathrm{mL}$ concentration range were used to construct calibration curves, as quantitative recoveries were obtained with QC samples over the $300-1800 \mathrm{ng} / \mathrm{mL}$ concentration range. Linear relationships were established from olaparib standard solutions over the $200-2000 \mathrm{ng} / \mathrm{mL}$ concentration range, by plotting the olaparib peak area versus the corresponding concentration in $\mathrm{ng} / \mathrm{mL}$. Concentrations of all standards were back calculated from the olaparib peak area using the calibration curve in which they were included. In every case, deviations within \pm $15 \%$ of the nominal concentration were found $( \pm 20 \%$ at the LOQ, defined as the lowest concentration of the standard curve that can be measured with acceptable inter- and intra-day precisions and accuracies). The average regression parameters of the linear regression function $(n=4)$ were $\mathrm{y}=0.0050( \pm 0.0002) \mathrm{x}+0.41( \pm 0.13)$. All calibration curves had correlation coefficient $\left(\mathrm{r}^{2}\right)$ of 0.997 or better. For the method described herein, the LOD was defined as $50 \mathrm{ng} / \mathrm{mL}$. Visual evaluation of standards chromatograms on which olaparib could be reliably detected and distinguished from the background noise was chosen among other accepted approaches for the determination of the LOD. The sensitivity of the method was evaluated by LOQ determination. For the current assay, the LOQ for olaparib was set to $200 \mathrm{ng} / \mathrm{mL}$, the lowest concentration that could be measured with acceptable precisions and accuracies following international recommendations. Lower values of LOQ were previously reported for the quantitation of olaparib but only LC-MS/MS methods were employed for such measurements, in cell culture medium, plasma, and tumor and brain homogenates [19,20,22,23]. With a LOQ of $200 \mathrm{ng} / \mathrm{mL}$, the current HPLC-UV was sufficiently sensitive and was successfully applied to the intracellular olaparib level determination in SUM1315 cells after various treatment times. The $254 \mathrm{~nm}$ detection wavelength 
was indeed chosen during the method development as a compromise to obtain sufficient sensitivity while keeping an excellent selectivity.

\subsubsection{Precision and accuracy}

Precision and accuracy of the current method were evaluated using three QC samples at concentrations ranging from 300 to $1800 \mathrm{ng} / \mathrm{mL}$. Samples were processed in a quintuple analysis in four analytical runs on four separate days. Precision and accuracy values are summarized in Table 2. The within- and between-day precisions, expressed as RSD (\%), were below 11\% at all QC levels. Similarly, the within- and between-day accuracies, expressed as bias (\%), were below $13 \%$ at all QC levels. Precision and accuracy therefore met the $\pm 15 \%$ required for method validation by the U.S. Food and Drug Administration [28].

\subsubsection{Stability}

Stability studies were also performed to ensure good reproducibility of the method. The stability of olaparib in acetonitrile was evaluated in different conditions on high and low QC samples. Tested conditions included shortterm room temperature and long-term low temperature storage. For short-term stability, QC samples were thawed and kept for $24 \mathrm{~h}$ at room temperature before analysis. Long-term stability was evaluated after a 30-day storage period at $-20^{\circ} \mathrm{C}$. Finally, data obtained after three freeze-thaw cycles was also collected. Results are shown in Table 3. No variation higher that $8.4 \%$ or $3.2 \%$ of the initial measured concentration was found for QC-low and QC-high samples, respectively. Hence, these results indicated that no stability issue would be expected after short or long term storage of the samples for quantification studies

\section{Method applicability}

The described method was developed to assess the intracellular level of a PARP inhibitor in cultured cells. A suitable sensitivity for standard cell culture conditions was achieved while limiting the number of steps during the sample pretreatment, namely culture medium removal, repeated wash step in ice-cold PBS, solubilization and sonication in acetonitrile, centrifugation for precipitated proteins elimination, and PVDF membrane filtration. Notably, no time-consuming sample concentration by solvent evaporation and subsequent reconstitution step are required. The described method was then used to determine the intracellular concentration of olaparib, a clinically relevant PARP inhibitor, in SUM1315 breast cancer cells after a $50 \mu \mathrm{M}$ treatment. Olaparib was detected in cell samples and the corresponding peak was integrated for quantitative analysis (Fig. 4). Results, expressed in ng/total cell number, are shown in Figure 5. Olaparib level in cells increased significantly $(\mathrm{p}<0.05)$ from 1 to $3 \mathrm{~h}$, which is in accordance with previous results [14]. Therefore, the described method allowed the monitoring of intracellular level of olaparib over time. Such data will be of great interest when considering the optimization of PARP inhibitor-based treatment efficacy.

\section{Conclusion}

In the present report, a simple, selective and sensitive reversed-phase HPLC method with UV detection was developed for determining PARP inhibitors intracellular concentration, and validated according to 
internationally accepted recommendations for bioassay validation. Importantly, the equipment required is readily available and much less expensive than other quantitative techniques such as tandem mass spectrometry. The proposed method has also the advantages of being straightforward and rapid, so that it was successfully applied to the quantitation of intracellular PARP inhibitor olaparib in SUM1315 breast cancer cells at various times of treatment. This analytical method could be extended to other cell lines and other PARP inhibitors to obtain valuable data for the successful prediction of drug efficiency in cancer treatment.

\section{Conflict of Interest}

The authors declare that they have no conflict of interest.

\section{Funding}

This research did not receive any specific grant from funding agencies in the public, commercial, or not-for-profit sectors.

\section{References}

[1] O. Dizdar, C. Arslan, K. Altundag, Advances in PARP inhibitors for the treatment of breast cancer, Expert. Opin. Pharmacother. 16 (2015) 2751-2758.

[2] D.V. Ferraris, Evolution of poly(ADP-ribose) polymerase-1 (PARP-1) inhibitors. From concept to clinic, J. Med. Chem. 53 (2010) 4561-4584.

[3] B. Lupo, L. Trusolino, Inhibition of poly(ADP-ribosyl)ation in cancer: old and new paradigms revisited, Biochim. Biophys. Acta 1846 (2014) 201-215.

[4] V. Schreiber, F. Dantzer, J.C. Ame, G. de Murcia, Poly(ADP-ribose): novel functions for an old molecule, Nat. Rev. Mol. Cell. Biol. 7 (2006) 517-528.

[5] J.S. Brown, S.B. Kaye, T.A. Yap, PARP inhibitors: the race is on, Br. J. Cancer 114 (2016) 713-715.

[6] J. Murai, S.Y. Huang, B.B. Das, A. Renaud, Y. Zhang, J.H. Doroshow, Y. Pommier, Trapping of PARP1 and PARP2 by Clinical PARP Inhibitors, Cancer Res. 72 (2012) 5588-5599.

[7] S. Tangutoori, P. Baldwin, S. Sridhar, PARP inhibitors: A new era of targeted therapy, Maturitas 81 (2015) 5-9.

[8] S. Murata, C. Zhang, N. Finch, K. Zhang, L. Campo, E.K. Breuer, Predictors and Modulators of Synthetic Lethality: An Update on PARP Inhibitors and Personalized Medicine, Biomed. Res. Int. (2016) 2346585.

[9] E.D. Deeks, Olaparib: first global approval. Drugs 75 (2015) 231-40.

[10] M.J. O'Connor, Targeting the DNA Damage Response in Cancer, Mol. Cell. 60 (2015) 547-560.

[11] C.T. Dollery, Intracellular drug concentrations, Clin. Pharmacol. Ther. 93 (2013) 263-266.

[12] T. Fojo, S. Bates, Mechanisms of resistance to PARP inhibitors-three and counting, Cancer Discov. 3 (2013) 20-23.

[13] C.J. Lord, A. Ashworth, Mechanisms of resistance to therapies targeting BRCA-mutant cancers, Nat. Med. 19 (2013) 1381-1388. 
[14] R. Dufour, P. Daumar, E. Mounetou, C. Aubel, F. Kwiatkowski, C. Abrial, C. Vatoux, F. Penault-Llorca, M. Bamdad, BCRP and P-gp relay overexpression in triple negative basal-like breast cancer cell line: a prospective role in resistance to Olaparib, Sci. Rep. 5 (2015) 12670.

[15] Y. Zhou, C. Joubran, L. Miller-Vedam, V. Isabella, A. Nayar, S. Tentarelli, A. Miller, Thinking outside the "bug": a unique assay to measure intracellular drug penetration in gram-negative bacteria, Anal. Chem. 87 (2015) 3579-3584.

[16] L.M. Colletti, Y. Liu, G. Koev, P.L. Richardson, C.M. Chen, W. Kati, Methods to measure the intracellular concentration of unlabeled compounds within cultured cells using liquid chromatography/tandem mass spectrometry, Anal. Biochem. 383 (2008) 186-193.

[17] A. Mateus, P. Matsson, P. Artursson, Rapid measurement of intracellular unbound drug concentrations. Mol. Pharm. 10 (2013) 2467-2478.

[18] D. Wu, C. Wang, J. Yang, H. Wang, H. Han, A. Zhang, Y. Yang, Q. Li, Improving the Intracellular Drug Concentration in Lung Cancer Treatment through the Codelivery of Doxorubicin and miR-519c Mediated by Porous PLGA Microparticle, Mol. Pharm. 13 (2016) 3925-3933.

[19] S. Durmus, R.W. Sparidans, A. Van Esch, E. Wagenaar, J.H. Beijnen, A.H. Schinkel, Breast cancer resistance protein (BCRP/ABCG2) and P-glycoprotein (P-GP/ABCB1) restrict oral availability and brain accumulation of the PARP inhibitor rucaparib (AG-014699), Pharm. Res. 32 (2015) 37-46.

[20] C.M. Nijenhuis, L. Lucas, H. Rosing, J.H. Schellens, J.H. Beijnen JH, Development and validation of a high-performance liquid chromatography-tandem mass spectrometry assay quantifying olaparib in human plasma, J. Chromatogr. B 940 (2013)121-125.

[21] R.W. Sparidans, S. Durmus, A.H. Schinkel, J.H. Schellens, J.H. Beijnen, Liquid chromatography-tandem mass spectrometric assay for the PARP inhibitor rucaparib in plasma, J. Pharm. Biomed. Anal. 88 (2014) $626-629$.

[22] R.W. Sparidans, I. Martens, L.B. Valkenburg-Van Iersel, J. Den Hartigh, J.H. Schellens, J.H. Beijnen, Liquid chromatography-tandem mass spectrometric assay for the PARP-1 inhibitor olaparib in combination with the nitrogen mustard melphalan in human plasma, J. Chromatogr. B 879 (2011) 1851-1856.

[23] J. Roth, C.J. Peer, B. Mannargudi, H. Swaisland, J-M. Lee, E.C. Kohn, W.D. Figg, A, A sensitive and robust Ultra HPLC assay with tandem mass spectrometric detection for the quantitation of the PARP inhibitor olaparib (AZD2281) in human plasma for pharmacokinetic application, Chromatography 1 (2014) 82-95.

[24] L. Oplustilova, K. Wolanin, M. Mistrik, G. Korinkova, D. Simkova, J. Bouchal, R. Lenobel, J. Bartkova, A. Lau, M.J. O'Connor, J. Lukas, J. Bartek, Evaluation of candidate biomarkers to predict cancer cell sensitivity or resistance to PARP-1 inhibitor treatment, Cell cycle 11 (2012) 3837-3850.

[25] C. Bourgne, M. Bamdad, A. Janel, F. Libert, M.C. Gagnieu, C. Rapatel, P. Pigeon, S. Pereira, E. Hermet, A. Guerci, B. Pereira, P.C. Makhoul, A.J. Ansah, J.Y. Cahn, D. Guyotat, S. Trouillier, J. Berger, N. Boiret-Dupré, M.G. Berger, Measurement of imatinib uptake by flow cytometry. Cytometry A 81 (2012) 996-1004

[26] B. Deng, Z. Wang, J. Song, Y. Xiao, D. Chen, J. Huang, Analysis of doxorubicin uptake in single human leukemia K562 cells using capillary electrophoresis coupled with laser-induced fluorescence detection, Anal. Bioanal. Chem. 401 (2011) 2143-2152

[27] C. Dubois, R. Dufour, P. Daumar, C. Aubel, C. Szczepaniak, C. Blavignac, E. Mounetou, F. PenaultLlorca, M. Bamdad, Development and cytotoxic response of two proliferative MDA-MB-231 and nonproliferative SUM1315 three-dimensional cell culture models of triple-negative basal-like breast cancer cell lines, Oncotarget 8(56) (2017) 95316-9533

[28] U.S. Food and Drug Administration, Department of Health and Human Services, http://www.fda.gov/downloads/Drugs/. . ./Guidances/ucm070107.pdf, 2001 (accessed 12.05.17) 


\section{Figures}

Fig. 1 Chemical structure of the PARP inhibitor olaparib

Fig. 2 UV absorption spectrum of Olaparib (the black arrow indicates $254 \mathrm{~nm}$, the wavelength used to monitor olaparib)

Fig. 3 Typical HPLC chromatograms of a control cell sample (a), a blank sample (b), a standard solution of olaparib in acetonitrile (1800 ng/mL) (c), and a QC-high sample (d)

Fig. 4 Typical HPLC chromatogram of a cell sample treated with olaparib $50 \mu \mathrm{M}$ for $3 \mathrm{~h}$

Fig. 5 Intracellular olaparib concentrations in SUM1315 cells treated with $50 \mu \mathrm{M}$ olaparib for 1 and $3 \mathrm{~h}(n=3)$

\section{Tables}

Table 1 Recovery (\%) of olaparib

\begin{tabular}{lc}
\hline Concentration $(\mathrm{ng} / \mathrm{mL})$ & $\begin{array}{c}\text { Recovery } \\
(\%)\end{array}$ \\
\hline QC-low $(300 \mathrm{ng} / \mathrm{mL})$ & $99.0 \pm 8.5$ \\
QC-mid $(800 \mathrm{ng} / \mathrm{mL})$ & $99.7 \pm 3.7$ \\
QC-high $(1800 \mathrm{ng} / \mathrm{mL})$ & $101.2 \pm 3.3$ \\
\hline
\end{tabular}

a Mean \pm standard deviation, $n=12$

Table 2 Within and between day precision and accuracy of the HPLC analytical method, expressed as RSD and bias percentages, respectively

\begin{tabular}{|c|c|c|c|c|c|c|}
\hline \multirow{2}{*}{$\begin{array}{l}\text { Nominal concentration } \\
(\mathrm{ng} / \mathrm{mL})\end{array}$} & \multicolumn{3}{|l|}{ Within day ${ }^{\mathrm{a}}$} & \multicolumn{3}{|l|}{ Between day ${ }^{b}$} \\
\hline & $\begin{array}{l}\text { Experimental concentration } \\
(\mathrm{ng} / \mathrm{mL})\end{array}$ & $\begin{array}{l}\text { RSD } \\
(\%)\end{array}$ & $\begin{array}{l}\text { Bias } \\
(\%)\end{array}$ & $\begin{array}{l}\text { Experimental concentration } \\
(\mathrm{ng} / \mathrm{mL})\end{array}$ & $\begin{array}{l}\text { RSD } \\
(\%)\end{array}$ & $\begin{array}{l}\text { Bias } \\
(\%)\end{array}$ \\
\hline 300 & $315 \pm 18$ & 5.71 & 4.84 & $288 \pm 21$ & 7.18 & -4.06 \\
\hline 800 & $789 \pm 19$ & 2.40 & -1.38 & $765 \pm 45$ & 5.92 & -4.35 \\
\hline 1800 & $1737 \pm 34$ & 1.97 & -3.53 & $1762 \pm 101$ & 5.74 & -2.09 \\
\hline
\end{tabular}

\footnotetext{
${ }^{\text {a }}$ Mean \pm standard deviation, $n=5$

${ }^{\mathrm{b}}$ Mean \pm standard deviation, $n=20$
} 
Table 3 Stability of olaparib drug concentration in acetonitrile (percentages of the initial measured concentration)

\begin{tabular}{lcc}
\hline Condition & $\begin{array}{c}\text { QC-low } \\
(\%)\end{array}$ & $\begin{array}{c}\text { QC-high } \\
(\%)\end{array}$ \\
\hline 24 h at ambient & $108.4 \pm 7.5$ & $96.8 \pm 3.6$ \\
3 freeze/thaw cycles & $95.0 \pm 3.0$ & $101.3 \pm 1.7$ \\
1 month at $-20^{\circ} \mathrm{C}$ & $93.3 \pm 5.3$ & $97.0 \pm 1.8$ \\
\hline
\end{tabular}

${ }^{\text {a }}$ Mean \pm standard deviation, $n=4$ 


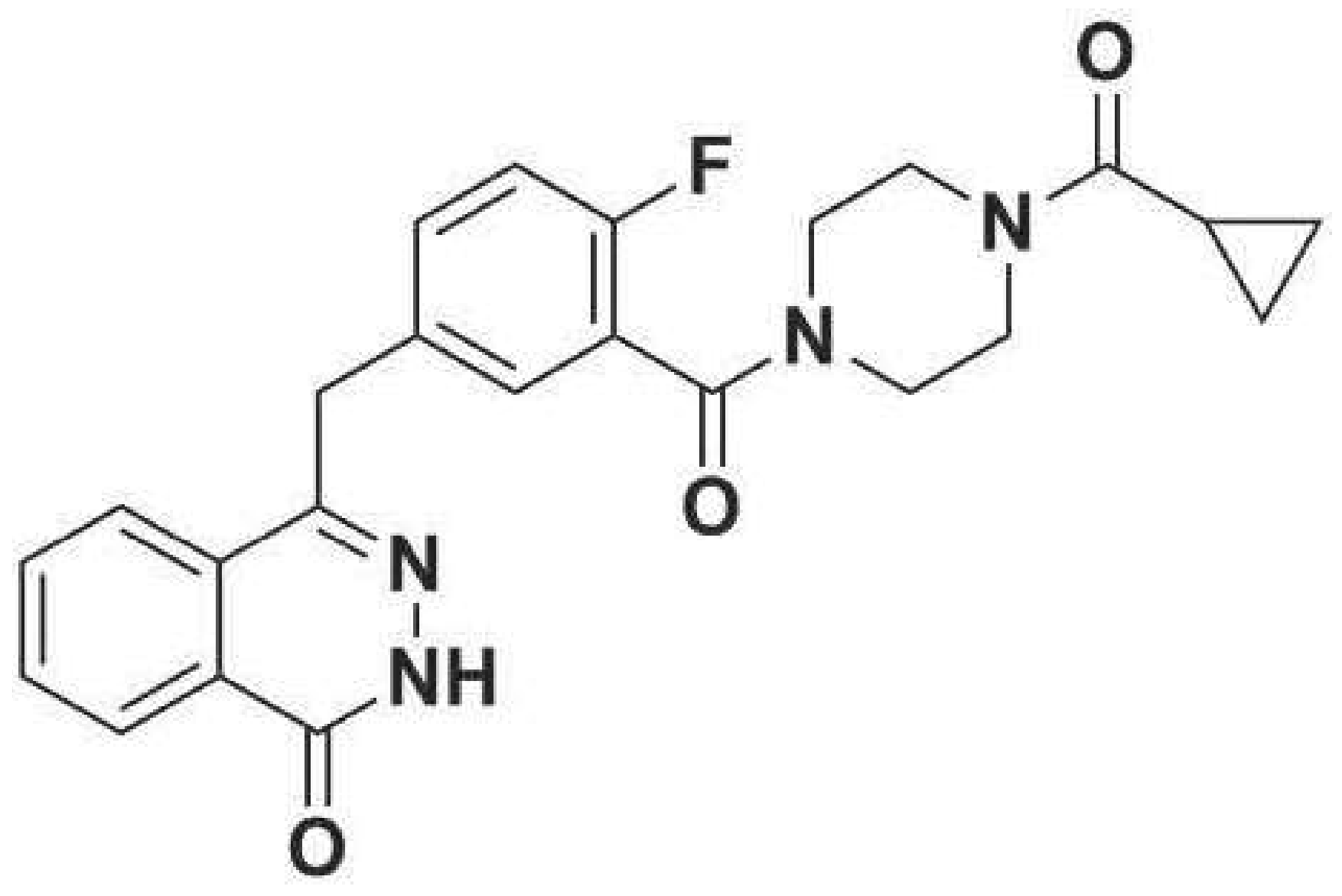




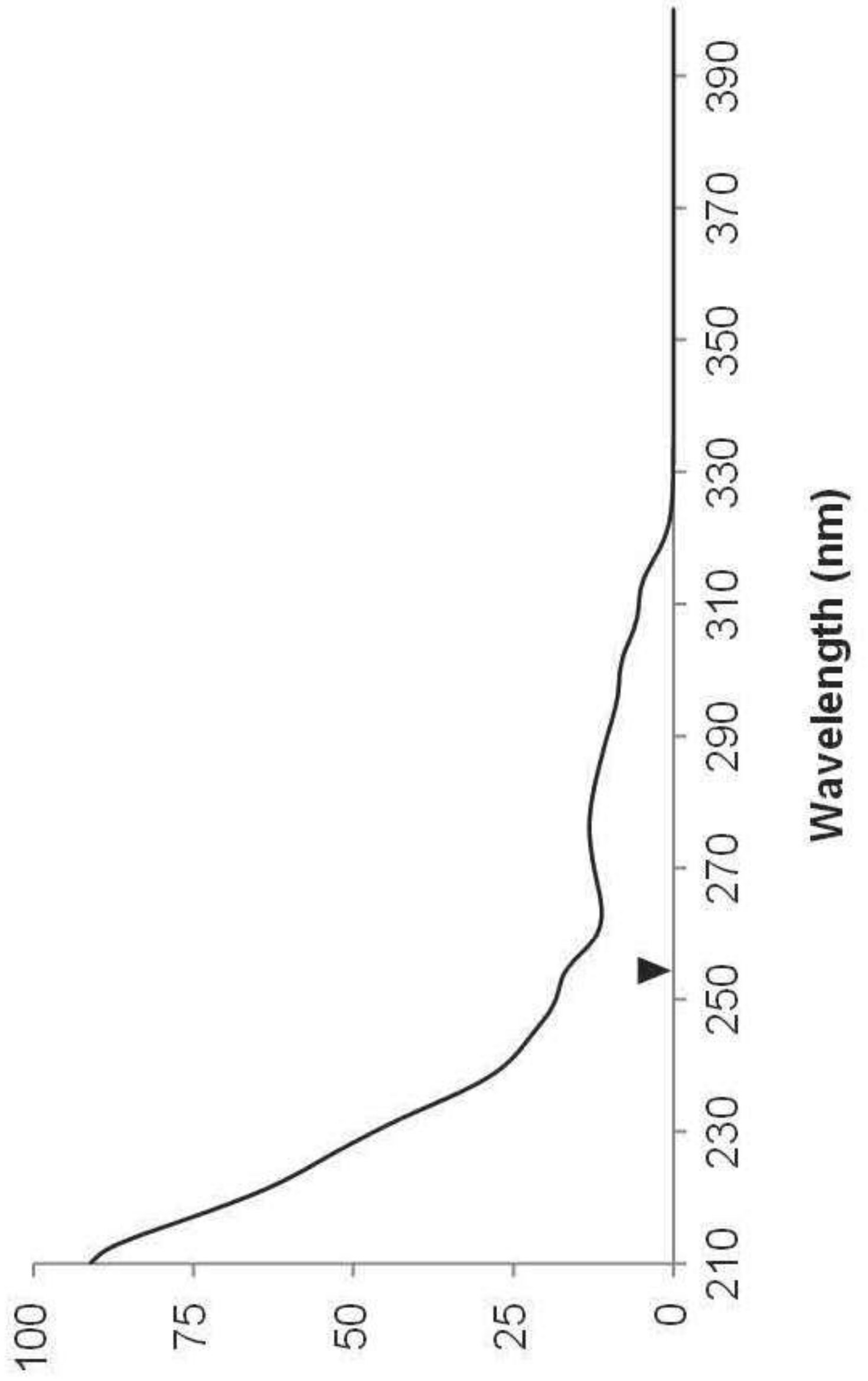

әэuequosqe $\Lambda$ pəs!|eu.ıoN 

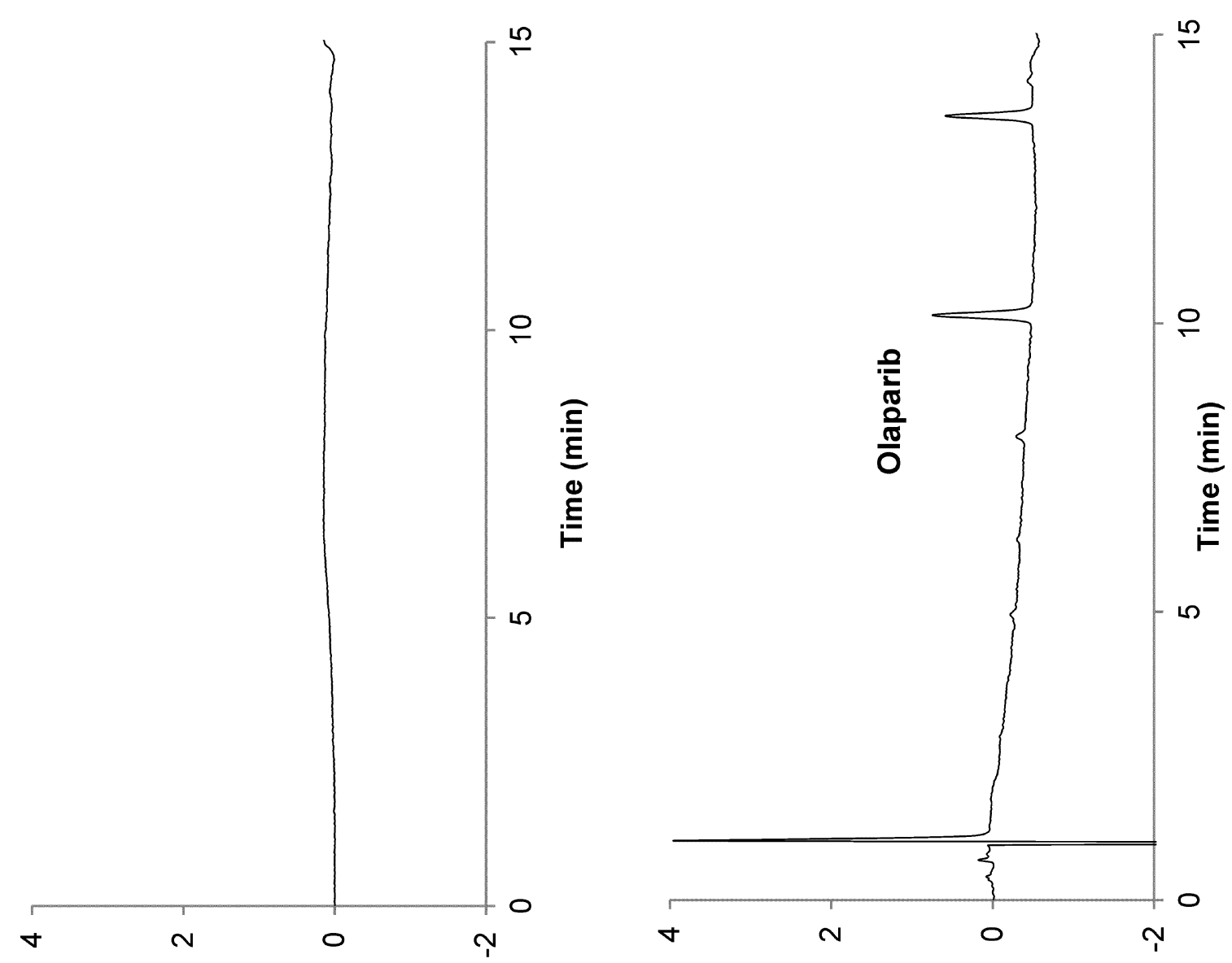

ㅇ

$n \forall m$

$\nabla$

ก
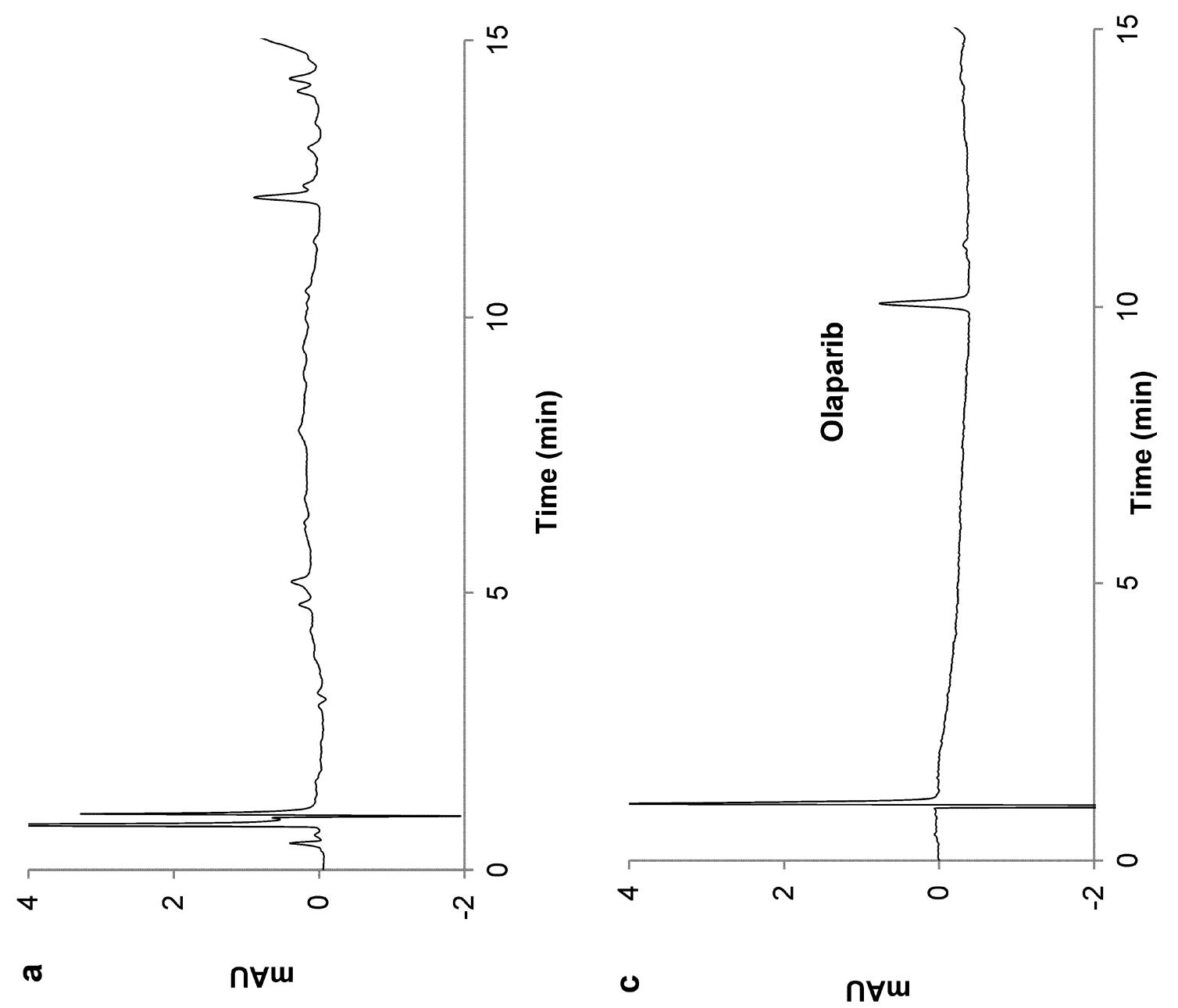

(ర

n $\forall \boldsymbol{m}$

n $\forall \mathbf{m}$ 


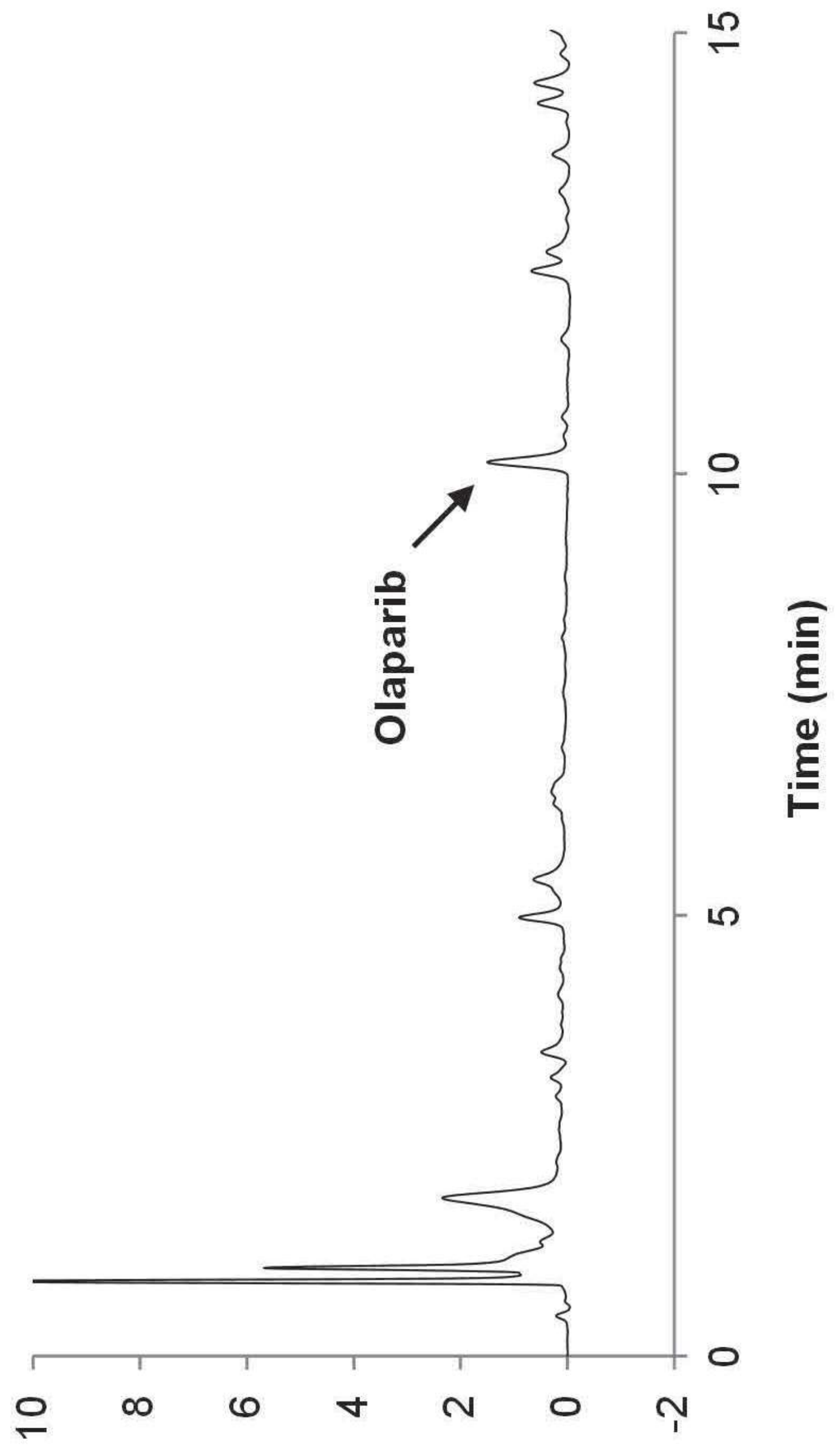

n $\forall \mathbf{u}$ 


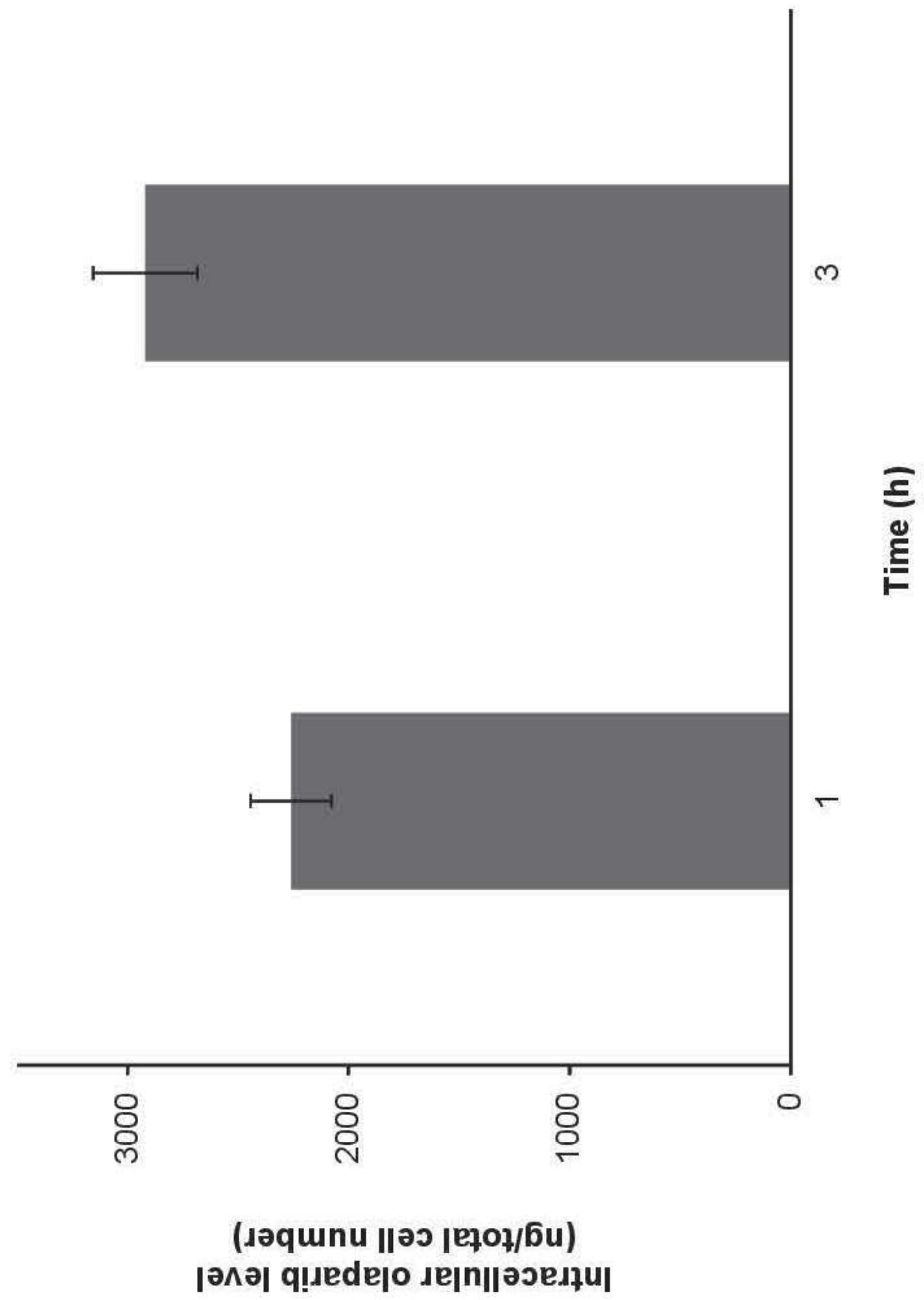

